The sclerotic was not involved by the growth (Fig. 1).

The mass of the tumour was composed of very broadly spindleshaped cells which showed a tendency in places to be arranged in coarse bundles (Fig. 2).

The nuclei were oval and well-formed; evidence of active mitosis was not observed.

Pigment was scattered throughout the growth in a somewhat uneven manner. The bulk of it was intra-cellular. I could not, however, be sure that none was extra-cellular.

In some places it was contained in globular cells; in other places it appeared to be contained in the long processes of branching cells.

The points in which this differed from the specimen previously reported were :-

(1) The pigmentation was less dense and less uniform.

(2) The arrangement of the cells was not quite so regular, and the large spheroidal cells, crowded with pigment, were much less conspicuous in this than in the former case.

There can, I think, be no doubt that this is an example of a melanoma of the choroid, benign in character, and probably congenital in origin.

\title{
THE REMOVAL OF FOREIGN BODIES BY MEANS OF A GIANT MAGNET.
}

BY

G. H. Pooley, F.R.C.S. SHEFFIELD.

In my earlier work with the giant magnet, I followed the technique of Haab. As my own experience has led me to modify very considerably the views I then held, and as I am convinced that I now get better results since the change in technique, I venture briefly to outline my views and my practice, giving my reasons.

Haab assumes that most foreign bodies are spindle-shaped (melon seed) and smooth. My experience is that they are more frequently irregularly triangular flakes with sharp angles, and that they are much inclined to turn broadside on when they meet any resistance. Most of the foreign bodies I have to deal with are from steel works, and are fresh from the fire and nearly aseptic.

When the foreign bodies are situated in or behind the lens my rules are :

1. Do not make any attempts to remove the foreign body before its situation has been carefully localized. Three or four days' wait makes no difference to the result. I have removed some foreign bodies after twenty years, others after ten years; and many in the second and third week, and later, with excellent results. 
2. Do not use a trial pull.

3. Do not pull on the ciliary body.

4. The foreign body should be removed from the nearest point.

5. Do not try to remove a foreign body embedded in the choroid.

My present practice, as modified by the result of my experience, is as follows :-

1. If the lens is badly wounded, so that it must become opaque and swell up, and the foreign body is situated immediately behind it, to remove the lens and then to remove the foreign body by pulling it into the anterior chamber.

2. If the lens is not wounded, or only slightly wounded, or for other reasons it is not desirable to remove the lens, and the foreign body is situated behind the lens, to remove the foreign body by means of a conjunctival incision through the sclera behind the ciliary body, usually behind the insertion of the extrinsic muscles. The technique is.-An antero-posterior conjunctival incision is made about $3 \mathrm{~mm}$. to one side of the site of the intended scleral incision and a temporary suture passed round the insertion of the nearest rectus muscle for fixation. A small parallel incision, about $4 \mathrm{~mm}$. or $5 \mathrm{~mm}$. long, is made in the sclera with a Graefe knife, and the point of a giant magnet placed upon or just introduced into this. If really necessary, a very small transverse section of the sclera may be added if the foreign body cannot be coaxed through the linear incision; the wound should close easily and not gape, for this reason the cross-section must be as small as will allow the foreign body to pass without bruising. The conjunctiva is sutured carefully at the end of the operation. I use alypin anæsthesia (retro-ocular injection) for all these cases.

Route No. 2 is the one which, I think, gives the best results. No. 1 is useful where the lens is going to give trouble very soon, as when there is a fairly large wound of the capsule in a man of over fifty years of age, whose lens will not easily absorb.

My reasons are as follows :-

1. A trial pull disturbs and clouds the vitreous unnecessarily and causes pain, which makes the patient less easy to control.

2. In all eye operations it is most essental to avoid bruising the tissues. The ciliary body is particularly intolerant of any bruising, if a foreign body has to be pulled through the suspensory ligament of the lens, the ciliary body is pulled on and the capsule of the lens is further damaged; the capsule may also be scratched; the iris is also bruised, and the inflammatory exudate which surrounds the foreign body, with any contained micro-organisms, is wiped off on the injured lens and bruised ciliary body, the result being :-

1. The eye is much more irritable and predisposed to attacks of cyclitis than is necessary.

2. The transparency of the lens is further imperilled. 
3. Already damaged tissues are again bruised, and their resistance to infection is further lowered.

Where a scleral route is adopted, the advantages are:

1. The operation is easier to perform, for the magnet is nearer, and so more efficient, and there is no resistance to the passage of the foreign body. The vitreous is disturbed as little as possible.

2. The inflammatory exudate may even come out with the foreign body.

3. The ciliary segment is not bruised.

4. A sutured conjunctival wound has much greater freedom from late infection than a corneal wound.

The disadvantages of the scleral wound is:-impaction of the choroid, retina, and vitreous in the wound. This very rarely occurs; I have found it in one or two cases where I have had to excise the eye eventually.

I claim the following advantages:-

(1) A larger percentage of eyes is saved. (2) A better visual acuity in the saved eye. (3) A shorter period of convalescence after operation.

\title{
THE TECHNIQUE OF THE HAAB AND SMALL MAGNETS IN THE EXTRACTION OF INTRA-OCULAR FOREIGN BODIES.
}

\author{
BY \\ Maurice, H. Whiting, and Charles Goulden, \\ CAPTAIN R.A.M.C. (TEMP.) LIEUTENANT R.A.M.C. (TEMP.)
}

DURING the past twelve months we have dealt at the Hospital to which we are attached with a great number of perforating wounds of the eye.

Many of these eyes contained magnetic foreign bodies.

Some idea of the number of such cases will be gathered from the fact that in July, 1916, alone, there were 30 cases in which magnetic foreign bodies were removed from within the globe; in one day, 5 such cases were operated upon, and more recently, 13 in one week.

With such a large amount of material, it has been our endeavour to work out some systematic plan of treatment applicable to the various conditions found ; and in the course of our operations we

- have elaborated many small points of technique, to overcome the varied difficulties encountered.

We are not aware that any detailed description of the use of the 